\title{
On the Controllability of Conformable Fractional Deterministic Control Systems in Finite Dimensional Spaces
}

\author{
Maher Jneid ${ }^{1}{ }^{1}$ and Muath Awadalla ${ }^{2}$ \\ ${ }^{1}$ Departement of Mathematics and Computer Science, Faculty of Science, Beirut Arab University, Beirut, Lebanon \\ ${ }^{2}$ Departement of Mathematics and Statistics, King Faisal University, Al-Ahsaa, Hufuf, Saudi Arabia \\ Correspondence should be addressed to Maher Jneid; m.jneid@bau.edu.lb
}

Received 7 October 2019; Accepted 23 December 2019; Published 10 March 2020

Academic Editor: Attila Gilányi

Copyright (c) 2020 Maher Jneid and Muath Awadalla. This is an open access article distributed under the Creative Commons Attribution License, which permits unrestricted use, distribution, and reproduction in any medium, provided the original work is properly cited.

\begin{abstract}
In this paper, we establish a set of convenient conditions of controllability for semilinear fractional finite dimensional control systems involving conformable fractional derivative. Indeed, sufficient conditions of controllability for a semilinear conformable fractional system are presented, assuming that the corresponding linear systems are controllable. The present method is based on conformable fractional exponential matrix, Gramian matrix, and the iterative technique. Two illustrated examples are carried out to establish the facility and efficiency of this technique.
\end{abstract}

\section{Introduction}

Controllability concepts have played a substantial role in several fields in engineering, control theory, and applied mathematics. In 1960, the controllability was first defined by Kalman [1] as a property of shifting the systems from any initial state value into any state value at a terminal time. This definition was divided into two notions: an exact and an approximate controllability which become more suitable for dealing with control systems in infinite dimensional spaces. The purpose of those notions is the existence of control systems which are approximately controllable, but are not exact (see [2]). In fact, the term exact controllability would refer to as a controllability which is the same as defined by Kalman. However, the definition of approximate controllability is determined by transferring the systems from any initial state value into some small neighbourhood of any point at terminal time in the state space. Later on, many researchers conducted pioneering studies in an attempt to obtain proper controllability conditions (exact and approximate) for the linear and nonlinear control systems (see, for example, [3-8] and the references cited therein).

Many problems in the real world can be modelled purely by fractional differential equations (for more details, refer to
$[9,10])$. This new calculus has pointedly attracted the mathematicians to focus clearly on revealing better results. The concept of controllability was extended to fractional control systems by various investigators. For instance, Sakthivel et al. [11] utilized fixed point approach to prove the controllability of nonlinear fractional systems. Vijayakumar et al. [12] obtained the controllability conditions for fractional integrodifferential neutral control systems with nonlocal conditions. Ma and Liu [13] employed analytic methods and resolvent operator to investigate controllability conditions and continuous dependence of a fractional neutral integrodifferential equation involving state-dependent delay. Jneid [14] derived sufficient conditions of approximate controllability for semilinear integrodifferential systems of fractional order with nonlocal conditions by using compact semigroup operator and Schauder fixedpoint theorem. Sakthivel et al. [15] studied the approximate controllability conditions for nonlinear fractional stochastic differential inclusions, providing that the corresponding linear part is approximately controllable. Chokkalingam and Baleanu [16] obtained a set of sufficient conditions for controllability for fractional functional integrodifferential systems involving the Caputo fractional derivative of order $\alpha \in(0,1]$ in Banach spaces. 
Previous works concerning controllability problems for fractional systems have been limited to Riemann, Liouville, and Caputo derivatives, while only one study concerning exact controllability involving conformable fractional derivative (CFD) as a definition of fractional derivative has been done by Jneid [17] till now. In this work, we aim at bringing up this kind of systems to the attention of investigators. Moreover, we derive controllability results for the semilinear conformable fractional system with initial condition $\xi$ :

$$
\left\{\begin{array}{l}
T_{0}^{q} x(t)=A x(t)+B u(t)+f(t, x(t), u(t)), \quad 0<t \leq \tau, 0<q \leq 1, \\
x(0)=\xi
\end{array}\right.
$$

where $T_{0}^{q}$ is a conformable fractional derivative $x \in C(0, \tau$; $\left.\mathbb{R}^{n}\right), u \in C\left(0, \tau ; \mathbb{R}^{m}\right), A \in \mathbb{R}^{n \times n}$ and $B \in \mathbb{R}^{n \times m}$, and $f:[0, \tau] \times$ $\mathbb{R}^{n} \times \mathbb{R}^{m} \longrightarrow \mathbb{R}^{n}$ is an appropriate nonlinear function.

The rest of this paper is divided into five sections. In Section 1, we provide needed fundamental information related to conformable fractional derivatives and we establish the mild solution of nonlinear systems involving conformable fractional derivative in terms of fractional exponential matrix by using Laplace transform. The controllability conditions for the linear systems are obtained in Section 3. In Section 4, an iterative analysis approach and controllability conditions are exhibited. We give two suitable examples to show the usefulness and effectiveness of this technique in Section 5. Finally, a short conclusion is given in Section 6 .

\section{Preliminaries}

Let $0<q \leq 1$ and $I=(0, \tau]$, through the entire article.

Definition 1 (see [18]). The CFD of a given function $f:(0, \infty) \longrightarrow \mathbb{R}$, at $t>0$ of order $q$ is given as

$$
T_{0}^{q} f(t)=\lim _{\epsilon \longrightarrow 0} \frac{f\left(t+\varepsilon t^{1-q}\right)-f(t)}{\epsilon},
$$

provided that the right side of this expression exists as a finite number.

Using this new definition of derivative, one can have the following properties which are similar to those of the classical derivative:

(a) For all constant $c, T_{0}^{q}(c)=0$.

(b) For all $s \in \mathbb{R}, T_{0}^{q}\left(t^{s}\right)=s t^{s-q}$.

(c) $T_{0}^{q}\left(e^{t^{q} / q}\right)=e^{t^{q} / q}$.

Definition 2 (see [18]). Given a function $f:(0, \infty) \longrightarrow \mathbb{R}$, the conformable fractional Laplace transform of $f$ at $t>0$ of order $q$ is given as

$$
\mathscr{T}_{0}^{q}\{f(t)\}(s)=F_{0}^{q}(s)=\int_{0}^{\infty} e^{-s t^{q} / q} f(t) t^{q-1} \mathrm{~d} t .
$$

Theorem 1 (see [18]). Given a differentiable function $f:(0, \infty) \longrightarrow \mathbb{R}$,

$$
\mathscr{T}_{0}^{q}\left\{T_{0}^{q} f(t)\right\}(s)=s F_{0}^{q}(s)-f(0) .
$$

Moreover,

$$
F_{0}^{q}(s)=\mathscr{T}\left\{f\left((q t)^{1 / q}\right)\right\}(s),
$$

where $\mathscr{T}$ is the classical Laplace transform.

Consider the conformable fractional system as follows:

$$
\left\{\begin{array}{l}
T_{0}^{q} x(t)=\mathscr{A} x(t)+f(t), \quad t \in I, \\
x(0)=\xi
\end{array}\right.
$$

where $T_{0}^{q}$ is the conformable fractional derivative operator, $x, f \in C\left(0, \tau ; \mathbb{R}^{n}\right)$, and $A$ is an $n \times n-$ matrix. Now, apply conformable fractional Laplace transform on system (6) to obtain

$$
X_{0}^{q}(s)=\xi \frac{1}{s I_{d}-A}+F_{0}^{q}(s) \frac{1}{s I_{d}-A},
$$

where $I_{d}$ is an $n \times n$-identity matrix. Utilizing the relation given in (5) and applying the inverse Laplace transform, one can get the solution of system (6) in this way:

$$
x(t)=e^{A\left(t^{q} / q\right)} \xi+\int_{0}^{t} e^{A\left(\left(t^{q} / q\right)-\left(s^{q} / q\right)\right)} f(s) s^{q-1} \mathrm{~d} s,
$$

where $e^{A\left(t^{q} / q\right)}=\sum_{k=0}^{\infty}\left(A^{k} t^{k q} / q^{k} k !\right)$ is called conformable fractional exponential matrix.

\section{Linear Control Systems}

Let us consider a linear conformable fractional control system that is described by

$$
\left\{\begin{array}{l}
T_{0}^{q} x(t)=A x(t)+B u(t), \quad t \in I, \\
x(0)=\xi
\end{array}\right.
$$

where $\xi \in \mathbb{R}^{n}$ is an initial condition, $T_{0}^{q}$ is the conformable fractional derivative operator, $x \in C\left(0, \tau ; \mathbb{R}^{n}\right), u \in C(0, \tau$; $\left.\mathbb{R}^{m}\right), A \in \mathbb{R}^{n \times n}$, and $B \in \mathbb{R}^{n \times m}$.

Lemma 1. The mild solution of system (9) on I in conformable fractional sense is given by

$$
x^{u}(t)=e^{A\left(t^{q} / q\right)} \xi+\int_{0}^{t} e^{A\left(\left(t^{q} / q\right)-\left(s^{q} / q\right)\right)} B u(s) s^{q-1} \mathrm{~d} s .
$$

Proof. This result follows simply from the forgoing section.

Denote the set of admissible controls by $U_{\mathrm{ad}}=C(0, \tau$; $\left.\mathbb{R}^{m}\right)$ and the reachable set of system (1) by

$$
R_{\xi, \tau}(f)=\left\{x^{u}(\tau) \in \mathbb{R}^{n} ; x^{u} \in C\left(0, \tau ; \mathbb{R}^{n}\right)\right\},
$$


where there exists $u \in U_{\text {ad }}$ such that $x^{u}$ satisfies system (1).

Definition 3. System (9) is said to be controllable on $I$ if $R_{\xi, \tau}(0)=\mathbb{R}^{n}$, for any $\xi \in \mathbb{R}^{n}$.

In other words, for any given $\xi, h \in \mathbb{R}^{n}$, system (9) can be reached to the intended state $h$ at terminal time $\tau$ from any initial state $\xi$. The controllability Gramian matrix for the linear system (9) is defined by

$$
{ }^{q} Q_{t-r}=\int_{r}^{t} s^{q-1} e^{A\left(s^{q} / q\right)} B B^{*} e^{A^{*}\left(s^{q} / q\right)} \mathrm{d} s, \quad 0 \leq r \leq t \leq \tau .
$$

For simplicity, we use ${ }^{q} Q_{t}$ and $Q_{t-r}$ instead of ${ }^{q} Q_{t-0}$, and ${ }^{q} Q_{t}$, respectively.

Theorem 2. System (9) is controllable on I if and only if $Q_{\tau}$ is invertible.

Proof. Let $Q_{\tau}$ be invertible. Then, $Q_{\tau}^{-1}$ exists and we can define a control $u$ as follows:

$$
u(t)=B^{*} e^{A^{*}\left(\left(\tau^{q} / q\right)-\left(\tau^{q / q}\right)\right)} Q_{\tau}^{-1}\left[h-e^{A\left(\tau^{q} / q\right)} \xi\right], \quad 0 \leq t \leq \tau,
$$

with $h$ and $\xi$ arbitrarily chosen from $\mathbb{R}^{n}$. Obviously, $u \in C\left(0, \tau ; \mathbb{R}^{m}\right)$.

Now, substituting this control into equation (10) at the terminal time $t=\tau$, we get

$$
\begin{aligned}
x^{u}(\tau)= & e^{A\left(t^{q} / q\right)} \xi+\int_{0}^{\tau} e^{A\left(\left(t^{q} / q\right)-\left(s^{q} / q\right)\right)} \\
& \cdot B B^{*} e^{A^{*}\left(\left(t^{q} / q\right)-\left(s^{q} / q\right)\right)} Q_{\tau}^{-1} \times\left[h-e^{A\left(t^{q} / q\right)} \xi\right] s^{q-1} \mathrm{~d} s \\
= & e^{A\left(t^{q} / q\right)} \xi+Q_{\tau} Q_{\tau}^{-1}\left[h-e^{A\left(t^{q} / q\right)} \xi\right] \\
= & h,
\end{aligned}
$$

which implies the controllability of system (9).

Conversely, let system (9) be controllable on $[0, \tau]$. Assume the contrary that ${ }^{q} Q_{\tau}$ is not invertible. Therefore, there exists a nonzero vector $y \in \mathbb{R}^{n}$ so that

$$
\begin{aligned}
y^{*} Q_{\tau} y & =0 \\
& =\int_{0}^{\tau} s^{q-1}\left\|y^{*} e^{A\left(\left(\tau^{q} / q\right)-\left(s^{q} / q\right)\right)} B\right\|^{2} \mathrm{~d} s .
\end{aligned}
$$

Hence,

$$
y^{*} e^{A\left(\left(\tau^{q} / q\right)-\left(s^{q} / q\right)\right)} B s^{q-1}=0, \quad \forall s \in[0, \tau] .
$$

Let $h=0$. Since system (9) is controllable, for every initial state value $\xi$, we can obtain a control $u$ leading the solution $x^{u}$ of (9) into 0 at terminal time $\tau$.

Select $\xi=-e^{A\left(\tau^{q / q)}\right.} y$. Thus,

$$
x^{u}(\tau)=e^{A\left(\tau^{q} / q\right)} \xi+\int_{0}^{\tau} e^{A\left(\left(\tau^{q} / q\right)-\left(s^{q} / q\right)\right)} B u(s) s^{q-1} \mathrm{~d} s=0 .
$$

This yields

$$
y=\int_{0}^{\tau} e^{A\left(\left(\tau^{q} / q\right)-\left(s^{q} / q\right)\right)} B u(s) s^{q-1} \mathrm{~d} s .
$$

Multiplying through by $y^{*}$ yields

$$
y^{*} y=\int_{0}^{\tau} y^{*} e^{A\left(\left(\tau^{q} / q\right)-\left(s^{q} / q\right)\right)} B u(s) s^{q-1} \mathrm{~d} s=0,
$$

which contradicts that $y \neq 0$. Hence, the controllability matrix ${ }^{q} Q_{\tau}$ is invertible.

\section{Semilinear Control Systems}

Let us consider a semilinear conformable fractional control system that is described by

$$
\left\{\begin{array}{l}
T_{0}^{q} x(t)=A x(t)+B u(t)+f(t, x(t), u(t)), \quad t \in I, \\
x(0)=\xi
\end{array}\right.
$$

where $A, B, \xi, x$, and $u f$ are defined as in the previous section.

For brevity, for any $\tau>0$, let $X=C\left(I ; \mathbb{R}^{n}\right)$. It is clear that the Cartesian product $X \times U_{\text {ad }}$ is a Banach space equipped with the norm

$$
\|(\cdot, \cdot)\|=\|(\cdot)\| X+\|(\cdot)\| U_{\text {ad }},
$$

where $\forall x \in X$ and $\forall u \in U_{\text {ad }}$, and

$$
\begin{aligned}
\|x\| X & =\max _{t \in I}\|x(t)\|, \\
\|u\| U_{\mathrm{ad}} & =\max _{t \in I}\|u(t)\| .
\end{aligned}
$$

Let us assume the following:

(A1) $f$ is bounded and satisfies Lipschitz continuity on $X \times U_{\text {ad }}$. That is, for every $t \in I, z_{1}, z_{2} \in \mathbb{R}^{n}$, and $v_{1}, v_{2} \in \mathbb{R}^{m}$, there exist $M>0$ and $N>0$ so that

$$
\begin{aligned}
\left\|f\left(t, z_{1}, v_{1}\right)\right\| & \leq M, \\
\left\|f\left(t, z_{1}, v_{1}\right)-f\left(t, z_{2}, v_{2}\right)\right\| & \leq N\left(\left\|z_{1}-z_{2}\right\|+\left\|v_{1}-v_{2}\right\|\right) .
\end{aligned}
$$

(A2) For every $\tau>0, Q_{\tau}$ is invertible.

Define the operator $F: X \times U_{\text {ad }} \longrightarrow X \times U_{\text {ad }}$ as

$$
F(x, u)(t)=(X(t), U(t)),
$$

where 


$$
\begin{aligned}
X(t)= & e^{A\left(t^{q} / q\right)} \xi+{ }^{q} Q_{t} e^{A\left(\left(\tau^{q} / q\right)-\left(t^{q} / q\right)\right)} Q_{\tau}^{-1}\left(h-e^{A\left(t^{q} / q\right)} \xi\right) \\
& +\int_{0}^{t} e^{A\left(\left(t^{q} / q\right)-\left(s^{q} / q\right)\right)} s^{q-1} f(s, x(s), u(s)) \mathrm{d} s, \\
& -\int_{0}^{t} Q_{t-s} e^{A^{*}\left(\left(\tau^{q} / q\right)-\left(t^{q} / q\right)\right)} Q_{\tau-s}^{-1} e^{A\left(\left(\tau^{q} / q\right)-\left(s^{q} / q\right)\right)} \\
& \cdot s^{q-1} f(s, x(s), u(s)) \mathrm{d} s, \\
U(t)= & B^{*} e^{A^{*}\left(\left(\tau^{q} / q\right)-\left(t^{q} / q\right)\right)}\left[Q_{\tau}^{-1} \times\left[h-e^{A\left(\tau^{q} / q\right)} \xi\right]\right. \\
& \left.-\int_{0}^{t} Q_{\tau-s}^{-1} s^{q-1} e^{A\left(\left(\tau^{q} / q\right)-\left(s^{q} / q\right)\right)} f(s, x(s), u(s))\right] .
\end{aligned}
$$

Introduce the iterative method as follows:

$$
\begin{aligned}
x_{0}^{u}(t)= & e^{A\left(t^{q} / q\right)} \xi+Q_{t} e^{A\left(\left(\tau^{q} / q\right)-\left(t^{q} / q\right)\right)} Q_{\tau}^{-1}\left(h-e^{A\left(\tau^{q} / q\right)} \xi\right), \\
x_{n+1}^{u}(t)= & x_{0}(t)+\int_{0}^{t} e^{A\left(\left(t^{q} / q\right)-\left(s^{q} / q\right)\right)} s^{q-1} \\
& \cdot f\left(s, x_{n}(s), u_{n}(s)\right) \mathrm{d} s \\
& -\int_{0}^{t} Q_{t-s} e^{A^{*}\left(\left(\tau^{q} / q\right)-\left(t^{q} / q\right)\right)} Q_{\tau-s}^{-1} e^{A\left(\left(t^{q} / q\right)-\left(s^{q} / q\right)\right)} s^{q-1} \\
& \cdot f\left(s, x_{n}(s), u_{n}(s)\right) \mathrm{d} s, \\
u_{0}(t)= & B^{*} e^{A^{*}\left(\left(\tau^{q} / q\right)-\left(t^{q} / q\right)\right)} Q_{\tau}^{-1} \times\left[h-e^{A\left(\tau^{q} / q\right)} \xi\right] \\
u_{n+1}(t)= & u_{0}(t)-B^{*} e^{A^{*}\left(\left(\tau^{q} / q\right)-\left(t^{q} / q\right)\right)} \\
& \times \int_{0}^{t} Q_{\tau-s}^{-1} s^{q-1} e^{A\left(\left(\tau^{q} / q\right)-\left(s^{q} / q\right)\right)} f\left(s, x_{n}(s), u_{n}(s)\right) .
\end{aligned}
$$

Denote $\Phi_{n}(t)=\left(x_{n}(t), u_{n}(t)\right.$ for all $t \in I$ and $n=0,1,2, \ldots$

$$
\begin{aligned}
P_{1} & =\max _{t \in I}\left\|t^{q-1} e^{A\left(\left(\tau^{q} / q\right)-\left(t^{q} / q\right)\right)}\right\|, \\
P_{2} & =\max _{t \in I}\left\|B^{*} e^{A^{*}\left(\left(\tau^{q} / q\right)-\left(t^{q} / q\right)\right)}\right\|, \\
P_{3} & =\max _{t \in I}\left\|e^{A^{*}\left(\left(\tau^{q} / q\right)-\left(t^{q} / q\right)\right)}\right\|, \\
\gamma & =\max _{t \in I}\left\|Q_{t}^{-1}\right\| .
\end{aligned}
$$

Lemma 2. Let the assumptions (A1) and (A2) hold true and let $n \geq 1$. Then,

$$
\left\|\Phi_{n+1}-\Phi_{n}\right\| \leq L_{1}^{n} L_{2}^{n} \frac{\tau^{n+1}}{n+1 !}
$$

where

$$
\begin{aligned}
& L_{1}=M P_{1}\left[1+\frac{P_{2}}{\gamma}+\frac{\left\|Q_{\tau}\right\| P_{3}}{\gamma}\right], \\
& L_{2}=P_{1} N\left[1+\frac{\left\|Q_{\tau}\right\| P_{3}}{\gamma}\right]+\frac{P_{1} P_{2} M}{\gamma} .
\end{aligned}
$$

Proof. Let us start to estimate $\left\|\Phi_{1}-\Phi_{0}\right\|$. By the definition of norm, we have

$$
\left\|\Phi_{1}-\Phi_{0}\right\|=\max _{t \in I}\left\|x_{1}(t)-x_{0}(t)\right\|
$$

$$
+\max _{t \in I}\left\|u_{1}(t)-u_{0}(t)\right\|
$$

$$
\begin{aligned}
\left\|x_{1}^{u}(t)-x_{0}^{u}(t)\right\|= & \| \int_{0}^{t} e^{A\left(\left(t^{q} / q\right)-\left(s^{q} / q\right)\right)} s^{q-1} \\
& \cdot f\left(s, x_{0}(s), u_{0}(s)\right) \mathrm{d} s \\
& -\int_{0}^{t} Q_{t-s} e^{A^{*}\left(\left(\tau^{q} / q\right)-\left(t^{q} / q\right)\right)} \\
& \cdot Q_{\tau-s}^{-1} e^{A\left(\left(\tau^{q} / q\right)-\left(s^{q} / q\right)\right)} s^{q-1} \\
& \cdot f\left(s, x_{0}(s), u_{0}(s)\right) \mathrm{d} s \| \\
\leq & {\left[P_{1} M+\frac{\left\|Q_{\tau}\right\| P_{3} P_{1} M}{\gamma}\right] t . }
\end{aligned}
$$

Similarly,

$$
\begin{aligned}
\left\|u_{1}(t)-u_{0}(t)\right\|= & \| t^{1-q} B^{*} e^{A^{*}\left(\left(\tau^{q} / q\right)-\left(t^{q} / q\right)\right)} \\
& \cdot \int_{0}^{t} Q_{\tau-s}^{-1} s^{q-1} e^{A\left(\left(\tau^{q} / q\right)-\left(s^{q} / q\right)\right)} \\
& \cdot f\left(s, x_{0}(s), u_{0}(s)\right) \| \\
\leq & \frac{P_{2} P_{1} M}{\gamma} t .
\end{aligned}
$$

Combining (32) and (33), we obtain

$$
\left\|\Phi_{1}(t)-\Phi_{0}(t)\right\| \leq L_{1} t,
$$

where 


$$
\begin{aligned}
L_{1}= & M P_{1}\left[1+\frac{P_{2}}{\gamma}+\frac{\left\|Q_{\tau}\right\| P_{3}}{\gamma}\right], \\
\left\|x_{2}^{u}(t)-x_{1}^{u}(t)\right\|= & \| \int_{0}^{t}\left(e^{A\left(\left(t^{q} / q\right)-\left(s^{q} / q\right)\right)}-Q_{t-s} e^{A^{*}\left(\left(\tau^{q} / q\right)-\left(t^{q} / q\right)\right)} Q_{\tau-s}^{-1} e^{A\left(\left(\tau^{q} / q\right)-\left(s^{q} / q\right)\right)}\right) \\
& \cdot\left(f\left(s, x_{2}(s), u_{2}(s)\right)-f\left(s, x_{1}(s), u_{1}(s)\right)\right) s^{q-1} \mathrm{~d} s \| \\
\leq & P_{1} N \int_{0}^{t}\left\|x_{2}(s)-x_{1}(s)\right\|+\left\|u_{2}(s)-u_{1}(s)\right\| \mathrm{d} s \\
& +\frac{\left\|Q_{\tau}\right\| P_{3} P_{1} N}{\gamma} \int_{0}^{t}\left\|x_{2}(s)-x_{1}(s)\right\|+\left\|u_{2}(s)-u_{1}(s)\right\| \mathrm{d} s \\
\leq & P_{1} N\left(1+\frac{\left\|Q_{\tau}\right\| P_{3}}{\gamma}\right) \int_{0}^{t}\left\|x_{1}(s)-x_{0}(s)\right\|+\left\|u_{1}(s)-u_{0}(s)\right\| \mathrm{d} s .
\end{aligned}
$$

In a similar manner, we get

$$
\begin{aligned}
\left\|u_{2}(t)-u_{1}(t)\right\|= & \|-B^{*} e^{A^{*}\left(\left(\tau^{q} / q\right)-\left(t^{q} / q\right)\right)} \\
& \cdot \int_{0}^{t} Q_{\tau-s}^{-1} s^{q-1} e^{A\left(\left(\tau^{q} / q\right)-\left(s^{q} / q\right)\right)} \\
& \cdot f\left(s, x_{1}(s), u_{1}(s)\right)+B^{*} e^{A^{*}\left(\left(\tau^{q} / q\right)-\left(t^{q} / q\right)\right)} \\
& \cdot \int_{0}^{t} Q_{\tau-s}^{-1} s^{q-1} e^{A\left(\left(\tau^{q} / q\right)-\left(s^{q} / q\right)\right)} \\
& \cdot f\left(s, x_{0}(s), u_{0}(s)\right) \| \\
\leq & \frac{P_{1} P_{2} M}{\gamma} \int_{0}^{t}\left\|x_{1}(s)-x_{0}(s)\right\| \\
& +\left\|u_{1}(s)-u_{0}(s)\right\| \mathrm{d} s .
\end{aligned}
$$

Combining (35) and (36) yields

$$
\left\|\Phi_{2}-\Phi_{1}\right\| \leq L_{2} \int_{0}^{t}\left\|x_{1}(s)-x_{0}(s)\right\|+\left\|u_{1}(s)-u_{0}(s)\right\| \mathrm{d} s,
$$

where

$$
L_{2}=P_{1} N\left[1+\frac{\left\|Q_{\tau}\right\| P_{3}}{\gamma}\right]+\frac{P_{1} P_{2} M}{\gamma} .
$$

Substituting (34) into (37), we obtain

$$
\begin{aligned}
\left\|\Phi_{2}-\Phi_{1}\right\| & \leq L_{2} \int_{0}^{t}\left\|\Phi_{1}(t)-\Phi_{0}(t)\right\| \mathrm{d} s, \\
& \leq L_{1} L_{2} \frac{t^{2}}{2 !} \leq L_{1} L_{2} \frac{\tau^{2}}{2 !} .
\end{aligned}
$$

Applying mathematical induction on $n \in \mathbb{N}$, we get the following estimation:

$$
\left\|\Phi_{n+1}-\Phi_{n}\right\| \leq L_{1}^{n} L_{2}^{n} \frac{\tau^{n+1}}{n+1 !}
$$

Lemma 3. Let assumptions (A1) and (A2) hold true. Then, the sequence of functions $\Phi_{n}$ defined as in (27) and (28) is uniformly convergent.

Proof. Identify $\Phi_{n+1}(t)=\Phi_{0}(t)+\left[\Phi_{1}(t)-\Phi_{0}(t)\right]+\Phi_{2}(t)-$ $\cdots+\left[\Phi_{n+1}(t)-\Phi_{n}(t)\right]$ as a partial sum of

$$
\Phi_{0}(t)+\sum_{k=0}^{\infty}\left[\Phi_{k+1}(t)-\Phi_{k}(t)\right] .
$$

Using the relation (40), we have

$$
\begin{aligned}
\left\|\Phi_{n+1}(t)\right\| \leq & \left\|\Phi_{0}(t)\right\|+\sum_{k=0}^{n}\left\|\Phi_{k+1}(t)-\Phi_{k}(t)\right\| \\
\leq & \left\|\Phi_{0}\right\|+L_{1} \tau+L_{1} L_{2} \frac{\tau^{2}}{2 !}+L_{1}^{2} L_{2} \frac{\tau^{3}}{3 !} \\
& +\cdots+L_{1}^{k} L_{2}^{k} \frac{\tau^{k+1}}{k+1 !} .
\end{aligned}
$$

It is easy to see that the sum in the equation (41) is convergent, and hence the sum in the equation (42) also converges as $n \longrightarrow \infty$. This implies that the sequence $\Phi_{n}$ converges since it is a partial sum of a convergent series. According to Weierstrass $M$-test, this convergence is uniform and hence the limit function, say, $\Phi$, for the sequence $\Phi_{n}$ is continuous.

Theorem 3. Let the assumptions (A1) and (A2) hold true. Then, the nonlinear map $F$ admits only one fixed point in $X \times U_{a d}$.

Proof. Thanks to Lemma 3 there is a pair $(x, u) \in X \times U_{\text {ad }}$ so that $\left(x_{n}, u_{n}\right) \longrightarrow(x, u)$ as $n \longrightarrow \infty$. Therefore, by taking the limit on both sides in (28) and (42), we see that the pair $(x, u)$ is a fixed point of $F$. Suppose that there are two distinct 
fixed points of $F$ say, $(x, u)$ and $(y, v)$ in $X \times U_{\text {ad }}$. Then, as in proof of Lemma 2 ,

$$
\|F(x, u)(t)-F(y, v)(t)\| \leq C \int_{0}^{t}\|F(x, u)(s)-F(y, v)(s)\| \mathrm{d} s,
$$

for some constant $C$.

Let $S(t)=\int_{0}^{t}\|F(x, u)(s)-F(y, v)(s)\| \mathrm{d} s$. It is clear that $S(0)=0$ and $\forall t \geq 0, \quad S(t) \geq 0$. Differentiating with respect to $t$, we obtain $S^{\prime}(t)=\|F(x, u)(t)-F(y, v)(t)\|$ and $S^{\prime}(t)-$ $C S(t) \leq 0$. Multiplying by exponential quantity $e^{-C t}$ gives

$$
\left[e^{-C t} S(t)\right]^{\prime} \leq 0
$$

Now, integrating through from zero to $t$ yields

$$
e^{-C t} S(t) \leq 0 \text {. }
$$

Since for all $t \geq 0, e^{-C t}>0$, then for all $t \geq 0, S(t) \leq 0$. Therefore, for all $t \geq 0, S(t)=0$, and consequently $S^{\prime}(t)=0$. Thus, $F(x, u)(t)=F(y, v)(t)$ for all $t \geq 0$. This contradicts the assumption that $(x, u)$ and $(y, v)$ are two distinct fixed points. This proof is completed.

Theorem 4. Let assumptions (A1) and (A2) hold true. Then, the semilinear control system (20) is controllable on I.

Proof. Fix $\xi \in \mathbb{R}^{n}$ and $h \in \mathbb{R}^{n}$. From Theorem 3, there is a unique mild solution $x \in X$ as defined in (25) which corresponds to a unique control $u \in U_{\text {ad }}$ as defined in (26). Hence, $x(\tau)=h$. Therefore, the semilinear system (20) is controllable on $I$.

\section{Examples}

Example 1. Consider the following conformable fractional control system:

$$
\left\{\begin{array}{l}
T_{0}^{q} x(t)=y(t)+u(t)+\sqrt{x^{2}(t)+5} \\
T_{0}^{q} y(t)=u(t)+\cos (u(t))
\end{array}\right.
$$

where $0 \leq t \leq 1,\left(x(0), y(0) \in \mathbb{R}^{2}, u \in C(0,1 ; \mathbb{R})\right.$. This system can be expressed in the following general form:

$$
T_{0}^{q} z(t)=A z(t)+B u(t)+f(t, z(t), u(t)),
$$

where

$$
\begin{aligned}
z & =\left[\begin{array}{l}
x \\
y
\end{array}\right], \\
A & =\left[\begin{array}{ll}
0 & 1 \\
0 & 0
\end{array}\right], \\
B & =\left[\begin{array}{l}
1 \\
1
\end{array}\right], \\
f(t, z(t), u(t)) & =\left[\begin{array}{l}
\sqrt{x^{2}(t)+5} \\
\cos (u(t))
\end{array}\right] .
\end{aligned}
$$

By simple calculations, we obtain

$$
e^{A\left(t^{q} / q\right)}=\left[\begin{array}{ll}
1 & \frac{t^{q}}{q} \\
& \\
0 & 1
\end{array}\right] .
$$

The controllability Gramian matrix is

$$
\begin{aligned}
Q_{1} & =\int_{0}^{1} s^{q-1} e^{A\left(s^{q} / q\right)} B B^{*} e^{A^{*}\left(s^{q} / q\right)} \mathrm{d} s, \\
& =\int_{0}^{1} s^{q-1}\left[\begin{array}{cc}
\left(1+\frac{s^{q}}{q}\right)^{2} & 1+\frac{s^{q}}{q} \\
1+\frac{s^{q}}{q} & 1
\end{array}\right] \mathrm{d} s .
\end{aligned}
$$

After a simple computation, we get

$$
Q_{1}=\left[\begin{array}{cc}
\frac{1}{q}+\frac{1}{q^{2}}+\frac{1}{3 q^{3}} & \frac{1}{q}+\frac{1}{2 q^{2}} \\
\frac{1}{q}+\frac{1}{2 q^{2}} & \frac{1}{q}
\end{array}\right] .
$$

Hence, for every $0<q \leq 1$, $\operatorname{det}\left(Q_{1}\right)=-\left(1 / q^{4}\right) \neq 0$, which means that $Q_{1}$ is invertible. In addition, the nonlinear function $f$ is bounded and satisfies Lipschitz condition with respect to $z$ and $u$ with the constant $N=1$; then, by Theorem 4 , the given control system (46) is controllable on $[0,1]$.

Example 2. Consider the following conformable fractional control system:

$$
\left\{\begin{array}{l}
T_{0}^{q} x(t)=x(t)+u(t)+\cos x \sin x \\
T_{0}^{q} y(t)=y(t)+v(t)+\sin (u(t)+v(t))
\end{array}\right.
$$

where $0 \leq t \leq 1,\left(x(0), y(0) \in \mathbb{R}^{2}, u, v \in C(0,1 ; \mathbb{R})\right.$. This system can be expressed in the following general form:

$$
T_{0}^{q} z(t)=A z(t)+B u(t)+f(t, z(t), w(t)),
$$

where

$$
\begin{aligned}
z & =\left[\begin{array}{l}
x \\
y
\end{array}\right], \\
A & =\left[\begin{array}{ll}
1 & 0 \\
0 & 1
\end{array}\right], \\
B & =\left[\begin{array}{ll}
1 & 0 \\
0 & 1
\end{array}\right], \\
f(t, z(t), w(t)) & =\left[\begin{array}{cc}
\cos x & \sin x \\
\sin (u(t)+v(t))
\end{array}\right] .
\end{aligned}
$$

By easy calculations, we have

$$
e^{A\left(t^{q} / q\right)}=\left[\begin{array}{cc}
e^{\left(t^{q} / q\right)} & 0 \\
0 & e^{\left(t^{q} / q\right)}
\end{array}\right]
$$

The controllability Gramian matrix is 


$$
\begin{aligned}
Q_{1} & =\int_{0}^{1} s^{q-1} e^{A\left(s^{q} / q\right)} B B^{*} e^{A^{*}\left(s^{q} / q\right)} \mathrm{d} s, \\
& =\int_{0}^{1} s^{q-1}\left[\begin{array}{cc}
e^{\left(2 s^{q} / q\right)} & 0 \\
0 & e^{\left(2 s^{q} / q\right)}
\end{array}\right] \mathrm{d} s .
\end{aligned}
$$

After a simple computation, we get

$$
Q_{1}=2 e^{2 / q}\left[\begin{array}{cc}
1 & 0 \\
0 & 1
\end{array}\right] \text {. }
$$

Hence, for every $0<q \leq 1, \operatorname{det}\left(Q_{1}\right)=2 e^{2 / q} \neq 0$, which means that $Q_{1}$ is invertible. In addition, the nonlinear function $f$ is bounded and satisfies Lipschitz condition with respect to $z$ and $w$ with the constant $N=1$; then, by Theorem 4, the given control system (52) is controllable on $[0,1]$.

\section{Conclusion}

In this work, the controllability conditions for semilinear conformable fractional deterministic systems are derived under a normal condition, that is, the associated linear system is controllable. The iterative technique is used here to construct a suitable sequence which is under some conditions uniformly convergent to a mild solution of the semilinear system. The present results show that this technique is very effective in finding the mild solution of semilinear control systems involving conformable fractional derivative. Finally, it should be mentioned that the result of this paper can be expanded to diverse kinds of conformable fractional systems in finite and infinite dimensional spaces as well.

\section{Data Availability}

No data were used to support this study.

\section{Conflicts of Interest}

The authors declare that they have no conflicts of interest.

\section{References}

[1] R. E. Kalman, "A new approach to linear filtering and prediction problems," Journal of Basic Engineering, vol. 82, no. 1, pp. 35-45, 1960.

[2] H. O. Fattorini, "Some remarks on complete controllability," SIAM Journal on Control, vol. 4, no. 4, pp. 686-694, 1966.

[3] A. E. Bashirov and M. Jneid, "On patial complete controllability of semilinear systems," Abstract and Applied Analysis, vol. 2013, Article ID 521052, 8 pages, 2013.

[4] A. E. Bashirov and M. Jneid, "Partial complete controllability of deterministic semilinear systems," Journal of Applied and Engineering Mathematics, vol. 4, pp. 216-225, 2014.

[5] A. E. Bashirov and N. I. Mahmudov, "On concepts of controllability for deterministic and stochastic systems," SIAM Journal on Control and Optimization, vol. 37, no. 6, pp. 1808-1821, 1999.

[6] H. Leiva and J. Uzcategui, "Exact controllability for semilinear difference equation and application," Journal of Difference Equations and Applications, vol. 14, no. 7, pp. 671-679, 2008.
[7] C. Guevara and H. Leiva, "Controllability of the impulsive semilinear heat equation with memory and delay," Journal of Dynamical and Control Systems, vol. 24, no. 1, pp. 1-11, 2016.

[8] H. Leiva, "Approximate controllability of semilinear impulsive evolution equations," Abstract and Applied Analysis, vol. 2015, Article ID 797439, 7 pages, 2015.

[9] I. Podlubny, Fractional Differential Equations, Academic Press, San Diego, CA, USA, 1999.

[10] V. Lakshmikantham and A. S. Vatsala, "Basic theory of fractional differential equations," Nonlinear Analysis: Theory, Methods \& Applications, vol. 69, no. 8, pp. 2677-2682, 2008.

[11] R. Sakthivel, N. I. Mahmudov, and J. J. Nieto, "Controllability for a class of fractional-order neutral evolution control systems," Applied Mathematics and Computation, vol. 218, no. 20, pp. 10334-10340, 2012.

[12] V. Vijayakumar, A. Selvakumar, and R. Murugesu, "Controllability for a class of fractional neutral integro-differential equations with unbounded delay," Applied Mathematics and Computation, vol. 232, pp. 303-312, 2014.

[13] H. Ma and B. Liu, "Exact controllability and continuous dependence of fractional neutral integro-differential equations with state-dependent delay," Acta Mathematica Scientia, vol. 37, no. 1, pp. 235-258, 2017.

[14] M. Jneid, “Approximate controllability of semilinear integrodifferential fractional control systems with nonlocal conditions," Applied Mathematical Sciences, vol. 11, no. 29, pp. 1441-1453, 2017.

[15] R. Sakthivel, Y. Ren, A. Debbouche, and N. I. Mahmudov, "Approximate controllability of fractional stochastic differential inclusions with nonlocal conditions," Applicable Analysis, vol. 95, no. 11, pp. 2361-2382, 2016.

[16] R. Chokkalingam and D. Baleanu, "On the controllability of fractional functional integrodifferential systems with an infinite delay in Banach spaces," Advances in Difference Equations, vol. 291, pp. 1-13, 2013.

[17] M. Jneid, "Exact controllability of semilinear control systems involving conformable fractional derivatives," AIP Conference Proceedings, vol. 2159, Article ID 030017, 2019.

[18] R. Khalil, M. Al Horani, A. Yousef, and M. Sababheh, "A new definition of fractional derivative," Journal of Computational and Applied Mathematics, vol. 264, pp. 65-70, 2014. 